\title{
Recente sismidade intraplaca no Domínio Noroeste do Ceará, região nordeste do Brasil
}

\author{
Paulo Henrique Sousa de Oliveira*1 - oliveiraphs@gmail.com \\ Joaquim Mendes Ferreira ${ }^{1}$, Francisco Hilário Rego Bezerra ${ }^{1}$, Aderson Farias do Nascimento ${ }^{1}$, Eduardo Alexandre Menezes \\ de Sousa ${ }^{1}$ \\ 1-PPGG/Universidade Federal do Rio Grande do Norte.
}

\section{Copyright 2014, SBGf - Sociedade Brasileira de Geofísica}

Este texto foi preparado para a apresentação no VI Simpósio Brasileiro de Geofísica, Porto Alegre, 14 a 16 de outubro de 2014. Seu conteúdo foi revisado pelo Comitê Técnico do VI SimBGf, mas não necessariamente representa a opinião da SBGf ou de seus associados. É proibida a reprodução total ou parcial deste material para propósitos comerciais sem prévia autorização da SBGf.

\section{RESUMO}

Além de sua importância geológica, estrutural e tectônica, o Domínio Noroeste do Ceará possui uma das principais atividades sísmicas ativas da região nordeste do Brasil. Com um histórico de ocorrência de sismos relatado desde o século XIX e uma numerosa e recente sismicidade (Ferreira et al., 1998; França et al., 2004; Oliveira et al., 2010 e Oliveira et al., 2011), este domínio tornou-se uma das principais regiões de estudos de sismologia do Laboratório Sismológico da UFRN. No início de janeiro de 2008, uma intensa atividade sísmica foi iniciada nas proximidades de Sobral com a ocorrência de um evento atingindo magnitude $4,2 \mathbf{m}_{\mathbf{b}}$, no dia 21 de maio de 2008, motivando a instalação de uma rede sismográfica local. Em agosto de 2009, uma atividade sísmica foi registrada nas proximidades da cidade de Santana do Acaraú, localizada na região noroeste do Ceará. Em novembro do mesmo ano, foi instalada uma rede sismográfica local, possibilitando o estudo da atividade sísmica revelada e constituiu de até 6 estações sismográficas digitais. Nesse estudo, foram analisados os sismos registrados, no mínimo, em três estações da rede sismográfica instalada na cidade de Santana do Acaraú, objetivando a determinação do diagrama de Wadati, de um modelo de velocidades e dos parâmetros hipocentrais.

\section{INTRODUÇÃO}

A cidade de Santana do Acaraú está localizada na porção noroeste do estado do Ceará, região nordeste do Brasil. Devido ao início de uma sismicidade nas proximidades dessa cidade em agosto de 2009, foi instalada uma rede sismográfica local com 6 estações digitais (rede $\mathrm{SA}$ ). A rede $\mathrm{SA}$ foi constituída por equipamentos pertencentes ao Pool de Equipamentos Geofísicos do Brasil (PEGBr), sediado no Observatório Nacional e com financiamento da PETROBRAS. A rede SA operou entre dezembro de 2009 e dezembro de 2010 com duas configurações. A primeira configuração foi mantida entre dezembro de 2009 e setembro de 2010 e a segunda, de outubro até dezembro de 2010. Antes da instalação da rede $S A$, outra rede sismográfica local operou recentemente na região noroeste do Ceará, nas proximidades de Sobral e da Serra da Meruoca (rede $\mathrm{SB})$. Os resultados da análise dos dados da rede SB, mostrados em Oliveira et al. (2010), contribuíram para um melhor entendimento da recente e numerosa sismicidade ocorrente na área estudada. Juntando-se a isso, a manifestação de várias áreas sísmicas e recentes eventos ocorridos no Domínio Noroeste do Ceará, temos uma das principais regiões de atividade sísmica ativa do nordeste do Brasil. Os resultados expostos nesse trabalho são provenientes das análises realizadas com os dados registrados pela rede $S A$, localizada na parte nordeste do Lineamento Transbrasiliano (LT). O LT é uma descontinuidade de magnitude continental, que se estende por quase $2700 \mathrm{~km}$ do nordeste até o sudeste do Brasil e atravessa a parte noroeste do Ceará, sendo designado nesse local como Lineamento Sobral - Pedro II, dividindo dois dos principais domínios geotectônicos da Província Borborema, o Domínio Noroeste do Ceará e o Domínio Ceará Central.

\section{ATIVIDADE RECENTE NO DOMÍNIO NOROESTE DO CEARÁ}

Ultimamente, as cidades mais abaladas por tremores de terra na região noroeste do Ceará são as cidades de Massapê, Santana do Acaraú e Sobral. Nos dias 04, 19 e 23 de abril de 2011, às 15h20min UTC, 01h19min UTC e 02h58min UTC, respectivamente, foram registrados três sismos nas estações SBSF e SBBR com magnitude até 2,7 $\mathbf{m}_{\mathbf{b}}$, sendo sentidos em Massapê e principalmente em Várzea da Cruz, localidade desta cidade. Segundo informações da Defesa Civil de Sobral, no dia 08 de agosto ocorreu um abalo sísmico que foi sentido principalmente na Serra de Meruoca e em Sobral. A análise dos dados registrados da estação NBLI (RSISNE), localizada em Livramento, no estado da Paraíba, mostraram que o sismo ocorreu às $13 \mathrm{~h} 53 \mathrm{~min}$ UTC, com magnitude preliminar estimada em 3,0 $\mathbf{m}_{\mathbf{b}}$. A estação NBLI está localizada aproximadamente a $570 \mathrm{~km}$ do epicentro deste evento.

No dia 17 de fevereiro de 2012, às 7h11min UTC, foi registrado nas estações SBBR e NBMO um tremor de magnitude 2,5 $\mathbf{m}_{\mathbf{b}}$ com epicentro na cidade de Barroquinha, situada a $100 \mathrm{~km}$. No dia 2 de agosto de 2012, dois eventos com magnitudes $2,9 \mathrm{mb}$ (7h14min UTC) e 2,2 mb (7h41min UTC), foram sentidos entre a serra da Meruoca e Sobral. Entre os dias 03 e 09 de novembro de 2012, foram registrados treze abalos sísmicos na Serra da Meruoca e nove abalos sísmicos na região de Santana do Acaraú, registrados nas estações NBMO, SBBR e NBPS (RSISNE). Um dos sismos (ocorrido no dia 03 de novembro) na Serra da Meruoca, às $07 \mathrm{~h} 30 \mathrm{~min}$ UTC, atingiu magnitude $1,8 \mathbf{m}_{\mathbf{b}}$. Entre os sismos ocorridos nas proximidades de Santana do Acaraú, dois atingiram magnitudes de $2,1 \mathbf{m}_{\mathbf{b}}$ e $1,9 \mathbf{m}_{\mathbf{b}}$, às 05h18min UTC e às 09h33min UTC, respectivamente. 
No ano de 2013 foram sentidos e registrados vários abalos sísmicos na região da serra da Meruoca e Sobral. Entre os principais, podemos citar: o sismo ocorrido no dia 17 de março que atingiu 2,9 $\mathbf{m}_{\mathbf{b}}$ (20h28min UTC); dois sismos registrados no dia 24 que atingiram $2,3 \mathbf{m}_{\mathbf{b}}$ (2h20min UTC) e 1,7 $\mathbf{m}_{\mathbf{b}}$ (2h21min UTC); dois tremores ocorridos no dia 27 de março com um deles atingindo 2,6 $\mathbf{m}_{\mathbf{b}}$ (15h41min UTC); um tremor ocorrido no dia 5 de maio alcançando 2,1 $\mathbf{m}_{\mathrm{b}}$ (7h20min UTC); um abalo sísmico ocorrido no dia 24 de outubro com 2,2 $\mathbf{m}_{\mathbf{b}}$ de magnitude (7h37min UTC) e tremor ocorrido no dia 25 de novembro com magnitude $2,2 \mathrm{~m}_{\mathrm{b}}$ registrado às $13 \mathrm{~h} 06 \mathrm{~min}$ UTC.

\section{METODOLOGIA E PROGRAMAS UTILIZADOS}

Analisamos sismos registrados, no mínimo, em três estações. Para realizar a leitura dos tempos de chegada e das polaridades das ondas $P$ e $S$, utilizamos 0 programa SAC (Tapley \& Tull, 1991). Os epicentros,

\section{RESULTADOS E DISCUSSÃO \\ Diagrama de Wadati}

Durante a operação da rede SA, 83 sismos foram registrados em três ou mais estações e foram selecionados para obter resultados. Desses 83 sismos, 66 foram registrados, no mínimo, em quatro estações, sendo utilizados para a execução do referido diagrama. Assim, foi selecionado um conjunto confiável de 55 sismos (Figura 1), e o coeficiente k obtido foi:

$$
\mathbf{k}=\frac{\mathbf{V}_{\mathbf{p}}}{\mathbf{V}_{\mathbf{S}}}=1,701 \pm 0,0038
$$

\section{Modelo de Velocidades e Hipocentros}

O conjunto consistente de 55 sismos obtido pelo diagrama de Wadati foi utilizado na determinação do conjunto de parâmetros com maior quantidade de sismos que tiveram as menores médias de erros. Inicialmente, variando $\mathbf{V}_{\mathbf{P}}$ de $5,0 \mathrm{Km} / \mathrm{s}$ a $6,4 \mathrm{Km} / \mathrm{s}$ e $\mathrm{k}=\mathbf{V}_{\mathrm{p}} / \mathbf{V}_{\mathbf{s}}$ de 1,60 a 1,74 (Bormann, 2002) e usando o programa HYPO71, foram observados muito conjuntos de sismos com $\mathbf{V}_{\mathbf{P}} / \mathbf{V}_{\mathbf{S}}$ próximos de 1,70 , valor bem próximo do obtido com o diagrama de Wadati. Então, fixando este valor, variando $\mathbf{V}_{\mathbf{P}}$ e testando os valores nas localizações hipocentrais, obtivemos menores erros verticais, horizontais e de RMS com os seguintes parâmetros: $V_{p}=6,00 \mathrm{~km} / \mathrm{s}$ e $\mathbf{k}=\mathbf{1 , 7 0}$. Esse modelo foi escolhido e será utilizado em todos os cálculos futuros. hipocentros e 0 modelo de velocidade foram determinados com o programa HYPO71 (Lee \& Lahr, 1975). Para determinar os parâmetros do modelo de crosta utilizado, inicialmente foi necessário encontrar um conjunto confiável de dados utilizando o diagrama de Wadati (Kissingler \& Engdahl, 1973), de forma conjunta. Sobre tais parâmetros, adotamos que a velocidade da onda $\mathrm{P}\left(\mathbf{V}_{\mathbf{P}}\right)$ e a razão $\mathbf{k}=\mathbf{V}_{\mathbf{p}} / \mathbf{V}_{\mathbf{S}}$ são consideradas constantes. As relocações hipocentrais foram realizadas com auxílio do programa HYPODD, que realiza relocações de sismos utilizando a técnica das duplasdiferenças (DD), levando vantagem do fato de que a separação hipocentral entre dois sismos é menor que a distância entre o evento e a estação e que as trajetórias dos raios percorridos entre a fonte sísmica e uma estação comum são similares ao longo de quase toda a trajetória (Waldhauser \& Ellsworth, 2000)

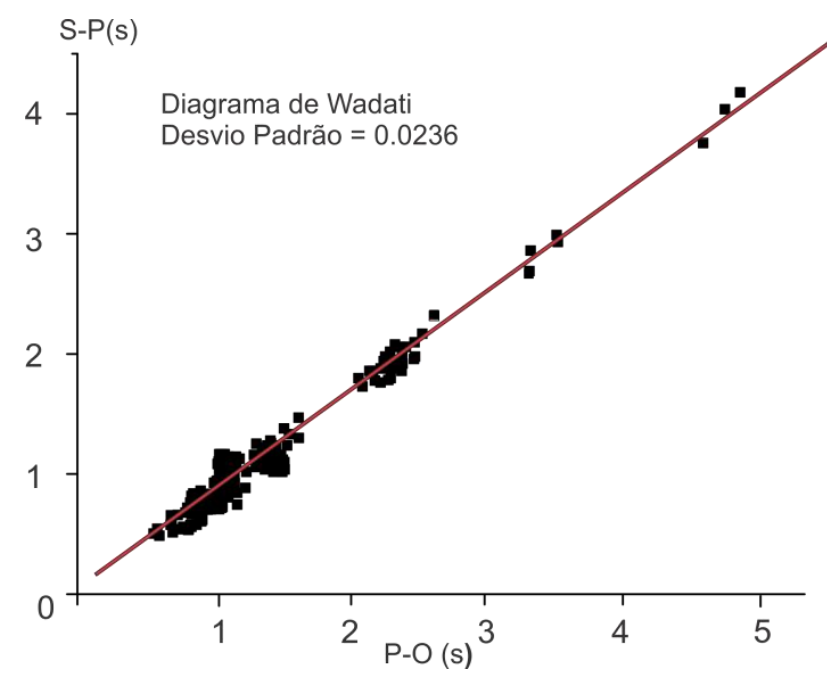

Figura 1 - Diagrama de Wadati, mostrando os sismos (quadrados pretos) constituintes do conjunto consistente de dados selecionados.

O modelo de crosta adotado foi um semi - espaço infinito, homogêneo e isotrópico, o qual fornece trajetórias de raios, provenientes das ondas $P$ e $S$, em forma de linhas retas. Esse modelo torna-se aceitável, pois a região sísmica está localizada sobre embasamento Pré Cambriano, o qual é constituído de rochas consolidadas e de baixa atenuação. Em geral, isso acarreta, nos sismogramas, chegadas das ondas $\mathrm{P}$ e $\mathrm{S}$ bem definidas (Figura 2). 


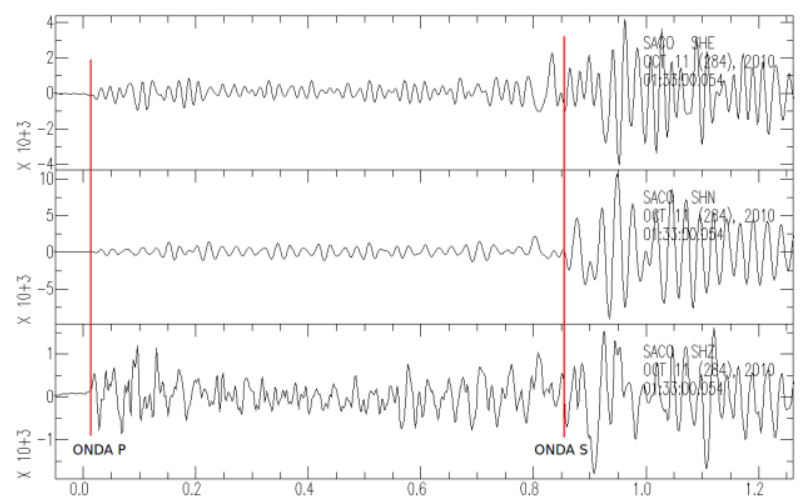

Figura 2 - Sismograma registrado na estação SACO de um evento ocorrido em 11 de outubro de 2010, às 01h33min (GMT), registrado em cinco estações da rede $S A . \quad O$ sismograma mostra P e S bem claras. SHE - componente E-W, SHN - componente N-S e SHZ - componente vertical.

A variação da atividade sísmica durante o período de operação em que foram registrados dados na rede SA está mostrada na figura 3, mostrando que o seu pico, com 45 sismos, foi observado no dia 5 de março de 2010.

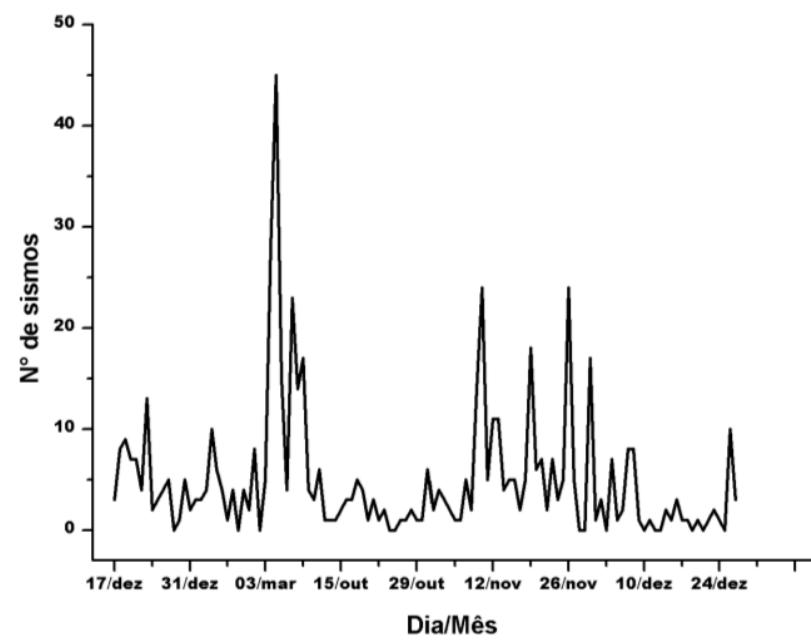

Figura 3 - Número de sismos por dia registrados durante toda a operação da rede SA. Apenas intervalos em que foram registrados dados.

\section{Primeira Configuração}

$\mathrm{Na}$ primeira configuração de rede utilizada foram registrados 38 sismos, no mínimo, em três estações. Na figura 5 são mostrados os epicentros calculados com auxílio do programa HYPO71.

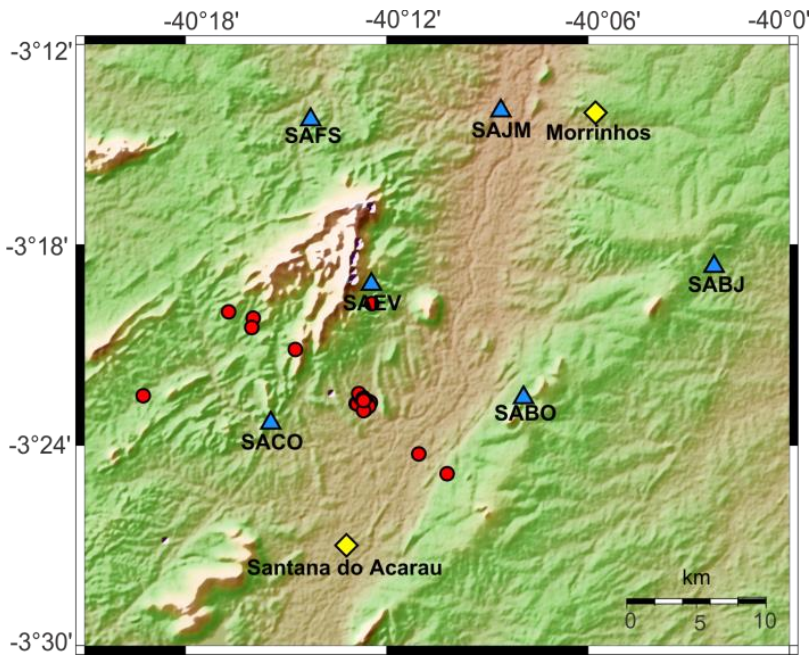

Figura 4 - Mapa epicentral dos 38 sismos registrados (círculos vermelhos), no mínimo, em três estações sismográficas, durante a primeira configuração da rede SA; com os seguintes critérios: $R M S \leq 0,04 s ; E R H \leq$ $0,35 \mathrm{~km} ; E R Z \leq 0,35 \mathrm{~km} ; N O \geq 6$.

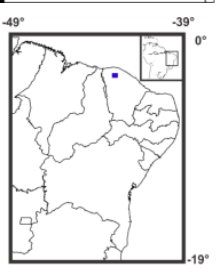

\section{Segunda Configuração}

Nessa configuração, foram registrados 66 sismos, no mínimo, em três estações. O mapa epicentral obtido está representado pela figura 5 .

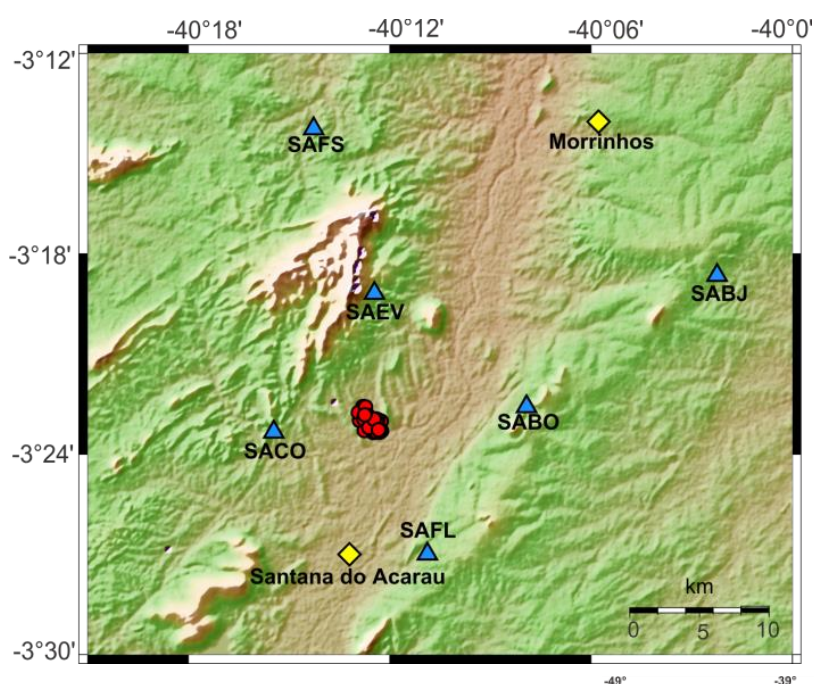

Figura 5 - Mapa epicentral dos 66 sismos registrados (círculos vermelhos), no mínimo, em três estações sismográficas, durante a segunda configuração da rede $S A$, utilizando os seguintes critérios: $R M S \leq 0,04 \mathrm{~s} ; E R H \leq$ $0,35 \mathrm{~km} ; E R Z \leq 0,35 \mathrm{~km} ; N O \geq 6$.

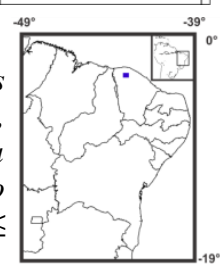

Na figura 4 os sismos tendem a se espalhar na direção NW-SE, apesar do aglomerado principal. O aglomerado mostrado na figura 5, não mostrou alinhamento preferencial em nenhuma direção. Nessa segunda configuração de rede, os erros verticais, horizontais e de 
RMS foram menores, comparado com aqueles calculados com os dados obtidos com a primeira configuração de rede.

\section{Relocação hipocentral}

Utilizamos dados de catálogo com fases das ondas $\mathrm{P}$ e $\mathrm{S}$ para executarmos o programa HYPODD. Durante a execução, foram testados vários parâmetros e obtemos sismos ligados e bem conectados. Após várias execuções com os seguintes parâmetros: MAXSEP $=5 \mathrm{~km}$, MAXDIST $=100 \mathrm{~km}$, foram relocados 56 sismos bem conectados, como mostrados na figura 6 . Os sismos relocados possuem certo alinhamento preferencial quase que igual ao alinhamento observado na figura 5 , a qual contém os sismos analisados na primeira configuração da rede SA.

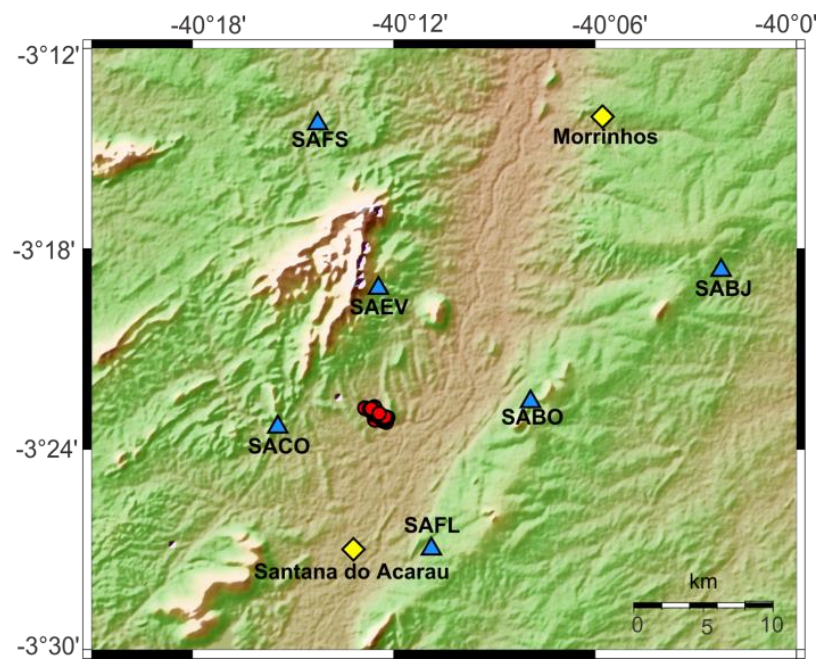

Figura 6 - Mapa de relocação hipocentral utilizando os sismos registrados, no mínimo, em 4 estações da rede $S A$

\section{CONCLUSÔES}

A sismicidade analisada nesse estudo encontra-se espalhada em várias áreas nas proximidades das cidades de Santana do Acaraú e Morrinhos. A análise detalhada da distribuição hipocentral e das polaridades das ondas $\mathrm{P}$ dos sismos locais registrados na rede $\mathrm{SA}$, evidenciam que há uma falha sismogênica na direção NW-SE. A sismicidade em ênfase aqui está bem próxima ao LT. No entanto, mais uma vez, não foi possível correlacionar a sismicidade com uma possível reativação, mostrando mais uma vez, que nem sempre é possível obter correlação entre sismicidade e falhas mapeadas. Atualmente, na área de estudo, não há evidência que possa concluir que o LT está reativado.

\section{AGRADECIMENTOS}

Os Autores agradecem aos técnicos do LabSls pela instalação, operação e manutenção da rede de estações localizada na região de Santana do Acaraú. Ao Dr. Marcelo Assumpção, do IAG/USP, pelo auxílio na análise dos dados. Ao INCT-ET pelos recursos financeiros e ao PEGBr pelos equipamentos de última geração utilizados. PHSO agradece à CAPES pela bolsa de doutorado.

\section{REFERÊNCIAS}

BEZERRA, F.H.R., do NASCIMENTO, A.F., FERREIRA, J.M., NOGUEIRA, F.C., FUCK, R.A., BRITO NEVES, B.B., SOUZA, M.O.L. 2011. Review of active faults in the Borborema Province, Intraplate South America Integration of seismological and paleoseismological data, Tectonophysics.

FERREIRA, J.M., OLIVEIRA, R.T., TAKEYA, M.K. \& ASSUMPÇÃO, M. 1998. Superposition of local and regional stress in northeast Brazil: evidence from focal mechanism around the Potiguar marginal basin. Geophys. J. Int., 134: 341-355.

KISSSLINGER, C. \& ENGDAHL, E.R. 1973. The interpretation of the Wadati diagram with relaxed assumptions. Bull.Seism.Soc.Am. 63: 1723-1736.

LEE, W.H.K. \& LAHR, J.C. 1975. HYPO71 (revised): a computer program for determining hypocenter, magnitude and first motion pattern of local earthquakes. U.S. Geol. Surv. Open File Rep. 75-311, 114 pp.

LEE, W.H.K, STEWART, S.W. 1981. Principles and Aplications of Microearthquake Networks. Academic Press, Inc (London), LTD.

OLIVEIRA, P.H.S., FERREIRA, J.M., do NASCIMENTO, A.F., BEZERRA, F.H.R., SOARES, J.E., FUCK, R.A., 2010. Estudo da Sismicidade na Região de Sobral - CE, NE do Brasil, em 2008. IV Simpósio da Sociedade Brasileira de Geofísica. Brasília - DF.

SANTOS, T.J.S., FETTER, A.H, HACKSPACHER, P.C., NOGUEIRA NETO, J.A. 2008. Neoproterozoic Tectonic and Magmatic Episodes in the NW Sector of Borborema Province, NE Brazil, During Assembly of Western Gondwana. Journal of South American Earth Sciences. 25: 271-284.

TAPLEY W.C. \& TULL. J.E. 1991. SAC-Seismic Analysis Code, USER MANUAL. Livermore National Laboratory, $413 \mathrm{pp}$.

WALDHAUSER, F., ELLSWORTH, W.L. 2000. A Double Difference Earthquakes Location Algorithm: Method and Application to the Northern Hayward Fault, California. Bulletim of the Seismological Society of America. v.90, n.6, pp.1353-1368. 